\title{
AVALIAÇÃO DAS CONDIÇÕES HIGIÊNICO- SANITÁRIAS EM RESTAURANTES COMERCIAIS EM UMA CIDADE DO NORTE DO RIO GRANDE DO SUL
}

Evaluation of hygienic-sanitary conditions in commercial restaurants in a city in the north of Rio Grande do Sul

\author{
Caroline Grosbelli ${ }^{1}$; Cilda Piccoli²; Jaqueline Sturmer³; Vivian Polachini Skzypek Zanardo ${ }^{4}$
}

${ }^{1}$ Acadêmica do Curso de Nutrição da Universidade Regional Integrada do Alto Uruguai e das Missões, URI Erechim.E-mail: carolgrosbelli007@gmail.com

${ }^{2}$ Nutricionista, Docente do Curso de Nutrição da Universidade Regional Integrada do Alto Uruguai e das Missões, URI Erechim, Mestra em Engenharia de Alimentos pela Universidade Regional Integrada e das Missões, URI Erechim.

${ }^{3}$ Nutricionista, Docente do Curso de Nutrição da Universidade Regional Integrada do Alto Uruguai e das Missões, URI Erechim, Mestra em Envelhecimento Humano pela Universidade de Passo Fundo, UPF.

${ }^{4}$ Nutricionista, Docente do Curso de Nutrição da Universidade Regional Integrada do Alto Uruguai e das Missões, URI Erechim, Doutora em Gerontologia Biomédica pelo Instituto de Geriatria e Gerontologia da PUCRS.

Data do recebimento: 28/10/2020 - Data do aceite: 22/02/2021

RESUMO: A realização das refeições fora de casa vem crescendo cada vez mais e os estabelecimentos mais procurados pela população são os restaurantes self-service. Os consumidores procuram por esse tipo de serviço, pois os mesmos oferecem uma grande variedade de alimentos e acessibilidade. O objetivo dessa pesquisa foi analisar as condições higiênico-sanitárias em restaurantes comercias em uma cidade do norte do Rio Grande do Sul. A pesquisa foi realizada em janeiro de 2020, em dois restaurantes comerciais escolhidos por conveniência, os quais foram denominados restaurante A e restaurante B. Para a coleta de dados foi realizada a verificação das condições higiênico-sanitárias dos restaurantes por meio da aplicação da Lista de Verificação de Boas Práticas para Serviços de Alimentação da Portaria 78/2009. 
A partir da aplicação da Lista de Verificação foram levantados os percentuais de conformidade dos estabelecimentos. O restaurante A obteve 45,6\% de adequação (classificado como ruim) e o restaurante B 56,4\% (classificado com regular). Sugere-se que os proprietários realizem adequações em seus estabelecimentos e forneçam aos seus funcionários cursos e capacitações com profissionais qualificados, para que os mesmos adquiram conhecimentos sobre os riscos alimentares que podem ser ocasionados devido à falta de cuidados na manipulação e armazenamento dos alimentos.

Palavras-chave: Restaurantes. Checklist. Manipulação de Alimentos.

\begin{abstract}
Eating out has been growing and growing, and the restaurants which are most used by the population are all-you-can-eat restaurants. Consumers are looking for this type of service, as they offer a wide variety of food and accessibility. The objective of this study was to analyze the hygienic-sanitary conditions in commercial restaurants in a city in the north of Rio Grande do Sul state. The research was carried out in January 2020 in two commercial restaurants chosen for convenience, which were called restaurant A and restaurant B. The data collection was performed through the verification of the restaurants hygiene and sanitary conditions based on the Checklist of Good Practices for Food Services of Ordinance 78/2009. With the application of the Checklist, the compliance percentages of the establishments were raised. Restaurant A obtained $45.64 \%$ of adequacy (rated as bad) and restaurant B $56.38 \%$ (rated as regular). It is suggested that the owners of the restaurants should make some adjustments in their establishments and provide their employees courses and training with qualified professionals, so that they can acquire knowledge about the food risks that may occur due to the lack of care in handling and storing food.
\end{abstract}

Keywords: Restaurants. Checklist. Food Handling.

\section{Introdução}

No Brasil tem crescido cada vez mais o número de refeições fora de casa. Alguns dos fatores que influenciaram nesse aumento foi a mudança do estilo de vida, a falta de tempo das pessoas para prepararem suas refeições e as transformações socioeconômicas.

Um dos estabelecimentos mais frequentados pelos brasileiros são os restaurantes self-service (MONTEIRO et al., 2014). Os restaurantes self-service são locais onde o próprio cliente se serve, as preparações ficam em balcões ao alcance do cliente (ABREU; TORRES, 2003; ANJOS et al., 2014). Esse tipo de serviço vem crescendo mundialmente, pois permite que o consumidor realize uma refeição completa e balanceada. Os consumidores procuram esse tipo de serviço, pois os mesmos oferecem uma grande variedade de alimentos, flexibilidade no horário das refeições e acessibilidade (JOMORI, 2006; ANJOS et al., 2014).

As Doenças Transmitidas por Alimentos (DTAs) são caracterizadas pelo consumo de água ou alimento contaminado por bactérias ou fungos e podem apresentar sintomas como 
náuseas, vômito e diarreia (BRASIL, 2015; NUNES et al., 2017).

Alguns fatores como a higiene do manipulador e dos utensílios utilizados na produção do alimento, a manipulação e exposição inadequada da matéria prima e do alimento pronto, a temperatura e a conservação imprópria dos produtos, são fatores de risco para a contaminação alimentar (BRASIL, 1993; TEIXEIRA et al., 1997; MAIA; MAIA, 2017).

Para ofertar alimentos seguros ao consumo é necessário que todos os utensílios, equipamentos, setores e áreas de produção estejam bem higienizados, assim como os manipuladores, que devem ter o conhecimento necessário para manipular os alimentos, sem que haja contaminações e para garantir uma alimentação segura (CARELLE; CÂNDIDO, 2014).

A segurança alimentar é de responsabilidade dos estabelecimentos que fornecem qualquer tipo de serviços de alimentação, sendo necessários cuidados nas etapas de coleta e manipulação dos alimentos, seleção, preparo, armazenamento, fracionamento, distribuição, exposição e venda dos mesmos (BRASIL, 2004; MELO; CRUZ; BESERRA, 2014).

O checklist é uma ferramenta que podemos utilizar para fazer uma breve avaliação das condições higiênico sanitárias dos estabelecimentos que produzem alimentos e preparações. Com esta avaliação podemos identificar os pontos que estão conformes e não conformes, a partir dos dados coletados. Daí pode-se criar ações corretivas e adequações para buscar eliminar ou reduzir riscos de contaminação dos alimentos e do consumidor (FIDELIS et al., 2010; COSTACURTA; PEREIRA; VASQUES, 2020).

As condições higiênico-sanitárias dos restaurantes comerciais estão adequadas de acordo com a legislação vigente? Estes restaurantes podem estar tanto adequados, quanto inadequados em relação a higiene sanitária necessária. Diante do exposto, o presente estudo teve como objetivo avaliar as condições higiênico-sanitárias em restaurantes comerciais, em uma cidade do Norte do Rio Grande do Sul.

\section{Material e Métodos}

Tratou-se de um estudo de cunho observacional, exploratório, descritivo e de caráter quantitativo e qualitativo, aprovado pelo Comitê de Ética em Pesquisa da Universidade Regional Integrada do Alto Uruguai e das Missões - URI - Câmpus Erechim - RS, sob o número CAAE - 24790819.6.0000.5351 e número do parecer 3.749.837.

$\mathrm{O}$ estudo foi realizado no mês de janeiro de 2020, em dois restaurantes comerciais escolhidos por conveniência, em uma cidade de pequeno porte e contendo poucos restaurantes, do norte do Rio Grande do Sul, sendo que os mesmos foram denominados como restaurante A e restaurante $\mathrm{B}$. A pesquisa teve início após a assinatura do Termo de autorização dos proprietários dos estabelecimentos. Ambos estabelecimentos não possuem o profissional nutricionista. Torna-se importante salientar a importância do profissional nutricionista nos estabelecimentos que fornecem alimentação para a população, pois os mesmos são capacitados para identificar fatores de risco à saúde, evitando e prevenindo doenças ao consumidor (KETZER, 2013; JUNIOR et al., 2020).

Para coleta de dados foi realizada a verificação das condições higiênico-sanitárias dos restaurantes, aplicando-se a Lista de Verificação em Boas Práticas para Serviços de Alimentação, conforme a Portaria $n^{\circ} 78$ de 30 de janeiro de 2009, vigente no estado do Rio Grande do Sul (RIO GRANDE DO SUL, 2009). 
A Lista de Verificação constitui um checklist composto por 153 critérios de avaliação categorizados em 12 grupos, distribuídos em verificações de vários aspectos do estabelecimento sendo eles: (1) Edificação, instalações, móveis e utensílios (n=34), (2) Higienização de instalações, equipamentos, móveis e utensílios $(n=17)$, (3) Controle integrado de pragas $(n=7),(4)$ Abastecimento de água ( $\mathrm{n}=9$ ), (5) Manejo de resíduos ( $\mathrm{n}=3),(6)$ Manipuladores $(\mathrm{n}=15),(7)$ Matérias primas, ingredientes e embalagens $(n=12),(8)$ Preparação do alimento $(n=26)$, (9) Armazenamento e transporte do alimento preparado $(n=6),(10)$ Exposição ao consumo do alimento preparado ( $\mathrm{n}=9)$, (11) Documentação e registro (n=4), (12) Responsabilidade técnica $(\mathrm{n}=7)$.

Cada critério contido na Lista de Verificação é dotado das opções "Sim", "Não" e "NA" (não aplicável), e foram assinalados com um " $\mathrm{x}$ " onde "Sim" corresponde a pontos de conformidade, quando os estabelecimentos estavam de acordo com os critérios referidos na legislação; Não corresponde à Não Conformidade, quando os itens analisados não estavam em conformidade e "NA", Não Aplicáveis, ou Não Notáveis quando os critérios não apresentaram nenhuma identificação com a realidade dos estabelecimentos. Desta forma os itens não aplicáveis foram desconsiderados e não foram computados no resultado do estudo.

A Lista de Verificação (checklist) foi aplicada e preenchida pela própria pesquisadora através da observação do local, das instalações físicas e questionamentos aos donos dos estabelecimentos. Os dados obtidos foram transformados em percentuais de critérios, atendidos através de "Sim" e "Não". Stangarlin; Serafim e Saccol (2016) classificam os estabelecimentos como: Excelente aqueles que atingem 91 a 100\% de adequação, Bom para os que atingem 70 a $90 \%$, Regular os que atingem 50 a $69 \%$, Ruim de 20 a $49 \%$ de adequação e Péssimo aqueles que atingem 0 a $19 \%$ de adequação dos requisitos. Para classificar os restaurantes foi utilizado Stangarlin; Serafim e Saccol (2016), pois a portaria 78/2009 não possui um modelo definido para classificar os Serviços de Alimentação, quanto à Implementação das Boas Práticas de fabricação.

Após a coleta de dados, os mesmos foram analisados por estatística descritiva e cálculos de proporção. Os resultados foram demonstrados através de tabela e figura, utilizando o programa Microsoft Office Excel ${ }^{\circledR} 2013$.

\section{Resultados e Discussões}

No presente estudo foram avaliados dois restaurantes comerciais através da Lista de Verificação, e a eles foram atribuídos percentuais. O percentual de adequações do restaurante A foi de $45,6 \%$ (classificado como ruim) e o restaurante $\mathrm{B} 56,4 \%$ (classificado como regular), de acordo com Stangarlin; Serafini e Saccol, 2016.

Resultado semelhante ao encontrado no restaurante A, deste estudo, foi verificado por Santos et al. (2015), que ao aplicarem o checklist disposto na Resolução RDC n²16/2004, em um restaurante universitário na cidade de Campina Grande, encontraram $37,3 \%$ de adequações.

Para o restaurante B resultados similares foram encontrados em alguns estudos. Stoffel e Barreto (2018) ao avaliarem as boas práticas de fabricação aplicando a lista de verificação conforme a Portaria ${ }^{\circ} 78 / 2009$, em um restaurante especializado em comida oriental na Serra Gaúcha - RS, encontraram $69,22 \%$ de adequação, isto classificou o restaurante avaliado como adequado com restrição, o que pode apresentar riscos à saúde do consumidor. Blanger; Nunes e Sant'Anna (2017) ao avaliarem 13 estabelecimentos de 
serviços de alimentação e sistema self-service na cidade de Encantado - RS, encontraram uma média de $67,42 \%$ de adequação de acordo com a portaria $\mathrm{n}^{\circ} 78 / 2009$, através da aplicação do guia de verificação ou checklist.

Na Tabela I estão demostrados os Percentuais de conformidade e quantidade total de itens avaliados, de acordo com a distribuição de cada categoria nos restaurantes A e B, segundo a Portaria ${ }^{\circ} 78 / 2009$.

Referente ao item 1- Edificação, Instalações, Equipamentos, Móveis e utensílios, o restaurante A obteve $50 \%$ de adequação e o restaurante B $58,8 \%$. Ambas as empresas não possuem separações entre diferentes atividades; o dimensionamento da edificação e das instalações não são compatíveis com todas as operações; não possuem portas com fechamento automático e não ocorre uma boa ventilação em nenhum dos locais.

Já no estudo de Santos et al. (2015) ao aplicarem o checklist disposto na Resolução RDC n²16/2004, em um restaurante universitário em Campina Grande, na Paraíba, obtiveram $29,1 \%$ de itens em conformidade, diferentemente do resultado encontrado no presente estudo. No restaurante universitário, o acesso à área interna não era independente. Era aberta e sem controle da entrada de pessoas que não trabalhavam no setor, o esgoto estava a céu aberto e havia presença de lixo exposto.

No item 2- Higienização de instalações, equipamentos, móveis e utensílios, o restaurante A obteve $35,3 \%$ de adequação e o restaurante B 52,9\%. A empresa A não utilizava produtos saneantes em seu estabelecimento, enquanto que a empresa B fazia o uso desses produtos. Além disso, a diluição, o tempo de contato e o modo de aplicação estavam de acordo com as informações do fabricante e se encontravam identificados e armazenados sem contato com os alimentos. Nenhuma das duas empresas possui registro de operações de limpeza das instalações e equipamentos quando não são realizados rotineiramente $\mathrm{e}$, também, não possuem um responsável capacitado para realizar a higienização.

Tabela I - Percentual de conformidades e quantidade total de itens avaliados nos restaurantes A e B

\begin{tabular}{|c|c|c|c|}
\hline Itens & $\begin{array}{l}\text { Resultados } \\
\quad(\%) \\
\text { Restauran- } \\
\text { te A }\end{array}$ & $\begin{array}{l}\text { Resulta- } \\
\text { do (\%) } \\
\text { Restau- } \\
\text { rante B }\end{array}$ & $\begin{array}{l}\text { Quanti- } \\
\text { dade total } \\
\text { de itens }\end{array}$ \\
\hline $\begin{array}{l}\text { 1- Edificação, } \\
\text { Instalações, } \\
\text { Equipamentos, } \\
\text { Móveis e } \\
\text { Utensílios }\end{array}$ & 50,0 & 58,8 & 34 \\
\hline $\begin{array}{l}\text { 2- Higienização } \\
\text { de Instalações, } \\
\text { Equipamentos, } \\
\text { Móveis e } \\
\text { Utensílios }\end{array}$ & 35,3 & 52,9 & 17 \\
\hline $\begin{array}{l}\text { 3- Controle } \\
\text { integrado de } \\
\text { pragas }\end{array}$ & 100 & 100 & 7 \\
\hline $\begin{array}{l}\text { 4- Abastecimento } \\
\text { de água }\end{array}$ & 22,2 & 77,8 & 9 \\
\hline 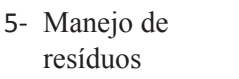 & 66,7 & 33,3 & 3 \\
\hline 6- Manipuladores & 66,7 & 60,0 & 15 \\
\hline $\begin{array}{l}\text { 7- Matérias } \\
\text { primas, } \\
\text { Ingredientes e } \\
\text { Embalagens }\end{array}$ & 41,7 & 58,3 & 12 \\
\hline $\begin{array}{l}\text { 8- Preparação do } \\
\text { alimento }\end{array}$ & 30,8 & 46,1 & 26 \\
\hline $\begin{array}{l}\text { 9- Armazenamen- } \\
\text { to e transporte } \\
\text { do alimento } \\
\text { preparado }\end{array}$ & ---- & ---- & 6 \\
\hline $\begin{array}{l}\text { 10-Exposição } \\
\text { ao consumo, } \\
\text { do alimento } \\
\text { preparado }\end{array}$ & 66,7 & 77,8 & 9 \\
\hline $\begin{array}{l}\text { 11-Documentação } \\
\text { e Registro }\end{array}$ & 50,0 & 50,0 & 4 \\
\hline $\begin{array}{l}\text { 12-Responsabili- } \\
\text { dade }\end{array}$ & 42,9 & 42,9 & 7 \\
\hline
\end{tabular}

Fonte: Dados da pesquisa, 2020. 
Em estudo de Lenz et al. (2019), ao aplicarem a Portaria 78/2009, em duas Unidades de Alimentação e Nutrição, em dois municípios do Vale do Taquari - RS, obtiveram 94,11\% de adequação, nesse quesito, na Unidades de Alimentação A e $86,66 \%$ na Unidade de Alimentação B, diferente do resultado encontrado no presente estudo. Apesar do resultado ter sido considerado bom, a Unidade B não possuía controle periódico de limpeza dos equipamentos e também não realizava a higienização da área de manuseio dos alimentos logo após o seu término.

No item 3- Controle integrado de pragas, ambos os restaurantes apresentaram 100\% de adequação. Stoffel e Barreto (2018), neste item, também obtiveram uma adequação de $100 \%$, após avaliarem um restaurante especializado em culinária oriental da Serra Gaúcha - RS e aplicarem a lista de verificação conforme a Portaria $n^{\circ} 78 / 2009$. Segundo a Portaria referida, deve existir ações eficazes e contínuas para a prevenção do controle de vetores e pragas urbanas, com o objetivo de impedir a atração, o abrigo, o acesso e a proliferação dos mesmos. Também é necessário que haja a existência de registros que comprovem o controle de vetores e pragas urbanas, tais como relatório de avaliação das medidas de controle realizadas por empresas especializadas (RIO GRANDE DO SUL, 2009).

No item 4 - Abastecimento de água, o restaurante A obteve 22,2\% de adequação e o restaurante B 77,8\%. A empresa A não utiliza fonte alternativa, já a empresa B utiliza e possui os registros que comprovam a higienização do reservatório, porém a higienização não é realizada no período máximo de seis meses.

Ao aplicarem um checklist embasado na Resolução RDC n 216/04, em uma lanchonete no município de Limoeiro do Norte CE, Maia e Maia (2017) encontraram 22\% de conformidades, resultado igual ao encontrado no restaurante $\mathrm{A}$. A lanchonete utilizava água potável para a higienização e preparo dos alimentos, porém foram encontradas inadequações que englobavam a higienização do reservatório de água.

No item 5- Manejo de resíduos, o restaurante $\mathrm{A}$ apresentou uma adequação de $66,7 \%$ e o restaurante B 33,3\%. Nenhuma das duas empresas possuem um local adequado para armazenar o lixo e a coleta de resíduos não ocorre com frequência. A empresa A possui coletores de resíduos de fácil higienização e transporte, dotados de tampas acionadas sem contato manual, íntegros, em quantidade necessária e com sacos plásticos. A empresa $\mathrm{B}$ possui coletores de fácil higienização e transporte, íntegros, em quantidades necessárias e com sacos plásticos, porém não são dotados de tampas sem contato manual.

Em um hospital de alta complexidade da cidade de Porto Alegre - RS, Silva et al. (2015) avaliaram a manipulação de alimentos em uma cozinha hospitalar com ênfase na segurança dos alimentos aplicando a lista de verificação da Portaria $n^{\circ} 78 / 2009$ e obtiveram $67 \%$ de adequações nesse quesito, resultado semelhante ao restaurante A.

No item 6- Manipuladores, o restaurante A apresentou 66,7\% de adequação e o restaurante B $60 \%$. Nenhuma das empresas possui cartazes orientando os funcionários sobre a correta higienização das mãos e demais hábitos de higiene. Os funcionários não são supervisionados e capacitados (tanto periodicamente como na admissão) e os visitantes não cumprem os requisitos de saúde e higiene estabelecidos.

Maciel et al. (2016) fizeram a verificação das boas práticas de fabricação em duas panificadoras localizadas na cidade de Marabá - PA e utilizaram um checklist com base nas resoluções RDC n²16/2004 e 275/2002. Resultado semelhante ao da presente pesquisa 
foi encontrado para o estabelecimento A, que obteve $53,8 \%$ de adequações. Entretanto para o estabelecimento B obteve-se um resultado diferente. Foi encontrado $23,08 \%$ de adequações. Em ambos os estabelecimentos os manipuladores não utilizavam sapatos fechados e de cor clara e nem máscaras; utilizavam adornos e esmaltes nas unhas.

No item 7- Matérias-primas, Ingredientes e Embalagens, o restaurante A apresentou $41,7 \%$ e o restaurante B $58,3 \%$ de adequação. Ambas as empresas não controlam a temperatura das matérias primas, não separam os alimentos de diferentes gêneros em um mesmo equipamento e não conferem a validade dos produtos quando recebidas. Na empresa A as matérias primas não são inspecionadas no recebimento e as mesmas não são armazenadas de forma correta.

$\mathrm{Na}$ cidade de Itaqui - RS, Silveira et al. (2015) avaliaram as condições higiênicas e boas práticas de manipulação em serviços de alimentação. Foram incluídos no estudo 24 serviços de alimentação sendo 10 restaurantes comercias, 7 lanchonetes, 5 padarias e 2 hotéis e utilizaram como instrumento de avaliação a lista de verificação da Portaria n $78 / 2009$. O resultado obtido nos hotéis nesse item foi de $58,33 \%$ de conformidades, igual ao encontrado no restaurante $\mathrm{B}$ neste estudo. Em nenhum dos hotéis era realizado o controle de temperatura dos alimentos, sendo que ambos possuíam termômetro.

No item 8- Preparação do alimento, o restaurante A apresentou $30,7 \%$ e o restaurante $\mathrm{B} 46,1 \%$. Ambas as empresas não deixam os produtos perecíveis em temperatura ambiente pelo tempo mínimo para a preparação do alimento, os alimentos que não são usados em sua totalidade não são acondicionados e identificados conforme a rotulagem, as temperaturas necessárias para o tratamento térmico de todo o alimento não são controladas e não são guardadas amostras de todos os alimentos.
Em estudo de Maia e Maia (2017), obtevese $29 \%$ de conformidades neste item. Alguns aspectos que estavam em inconformidade eram o modo de descongelamento e a falta do monitoramento da temperatura de armazenamento dos alimentos e também a falta de etiquetas para os alimentos preparados.

No item 9- Armazenamento e transporte do alimento preparado, os dois restaurantes obtiveram um resultado nulo, pois não transportam alimentos para outros estabelecimentos.

No trabalho de Santos et al. (2015) obteve-se os mesmos resultados da presente pesquisa, uma vez que o restaurante universitário avaliado pelos autores também não fazia armazenamento e transporte dos alimentos preparados, visto que, a quantidade de alimentos preparada era consumida em sua totalidade no próprio restaurante.

No item 10- Exposição ao consumo do alimento preparado, o restaurante A obteve $66,7 \%$ e o restaurante B $77,8 \%$ de adequação. Ambas as empresas não possuem o registro da temperatura do equipamento de exposição dos alimentos.

Neste item Silva et al. (2015) obtiveram $50 \%$ de adequações no Hospital de Alta Complexidade de Porto Alegre - RS, resultado inferior ao encontrado nesta pesquisa. As inadequações do local se devem à inexistência do registro de temperatura do equipamento de distribuição dos alimentos.

No item 11- Documentação e registro, ambos os restaurantes obtiveram $50 \%$ de adequação. Os dois estabelecimentos possuem Procedimentos Operacionais Padronizados (POPs) e o Manual de Boas Práticas, porém não são colocadas em prática as atividades propostas nos mesmos.

Ao avaliarem um restaurante universitário, Santos et al. (2015) obtiveram $25 \%$ de conformidades, resultado inferior ao encontrado na presente pesquisa. De acordo com 
a Portaria $\mathrm{n}^{\circ} 78 / 2009$, os estabelecimentos devem conter os POPs, com a sequência de execução e ações corretivas, especificando o cargo e ou função dos responsáveis pelas atividades. É necessário que os estabelecimentos disponham desses POPs e sejam disponíveis aos funcionários envolvidos e à autoridade sanitária e que implementem os Procedimentos Operacionais Padrões de higienização de instalações, equipamentos e móveis, controle integrado de vetores e pragas urbanas, higienização do reservatório e higiene e saúde dos manipuladores.

No item 12- Responsabilidade, ambos os restaurantes obtiveram um resultado de $42,9 \%$ de adequação. Em nenhum dos estabelecimentos são disponibilizados treinamentos sobre higiene pessoal, manipulação higiênica dos alimentos e doenças transmitidas por alimentos, sendo que os proprietários também não realizam tais treinamentos.

Resultado superior ao desta pesquisa foi encontrado por Maia e Maia (2017), que obtiveram $50 \%$ de conformidades em seu estudo. Na lanchonete avaliada não havia um responsável técnico capacitado e as funções que deveriam ser realizadas pelo responsável técnico eram desempenhadas pelo próprio proprietário da lanchonete.

Segundo a Portaria $n^{\circ} 78 / 2009$, o responsável pelas atividades de manipulação dos alimentos necessita ter comprovante da realização do Curso de Capacitação em Boas Práticas para Serviços de Alimentação, tendo sido abordado, no mínimo, contaminação de alimentos, doenças transmitidas por alimentos, manipulação higiênica dos alimentos e Boas Práticas. O responsável pela manipulação também deve sempre se atualizar com cursos e palestras, no mínimo, anualmente, e também promover treinamentos, no mínimo, anuais, para seus colaboradores.

\section{Considerações finais}

Através da aplicação da lista de verificação, foi possível avaliar que os dois restaurantes não estão totalmente de acordo com a legislação vigente. Com isso ambos os estabelecimentos podem oferecer riscos à saúde do consumidor. $\mathrm{O}$ restaurante $\mathrm{A}$ obteve um resultado inferior a $50 \%$, o que o classifica como ruim, podendo assim comprometer a qualidade dos alimentos e a segurança alimentar dos comensais. O restaurante B obteve $56,4 \%$ de adequações e foi classificado como regular. Se não ocorrer os devidos cuidados de higiene e na manipulação também pode oferecer riscos à segurança dos comensais.

Diante do exposto, sugere-se que os proprietários realizem adequações em seus estabelecimentos e forneçam aos seus funcionários cursos e capacitações com profissionais qualificados, para que os mesmos adquiram conhecimentos sobre os riscos alimentares que podem ser ocasionados devido à falta de higiene pessoal, dos cuidados quanto à manipulação e armazenamento dos alimentos e preparações, e que sejam capacitados para colocarem em prática as atividades dispostas no Manual de Boas Práticas. Tem-se como sugestão, também, contratar novos funcionários, para que seja designado para os mesmos, tarefas específicas referentes à limpeza ou até mesmo para supervisionar todas as etapas de produção e preparo dos alimentos, se certificando para que não ocorram falhas no processo de produção e acarretando problemas relacionados à alimentação. 


\section{REFERÊNCIAS}

ABREU, E. S.; TORRES, E. A. F. S. Restaurante "por quilo": vale o quanto pesa? Uma avaliação do padrão alimentar em restaurantes de São Paulo, SP. Nutrire: Revista da Sociedade Brasileira de Alimentação e Nutrição, v. 26, p. 7-22, jun. 2003.

ANJOS, C. M.; SANTANA, L. S.; SOUZA, T. C. B.; OLIVEIRA, L. C. Avaliação dos Fatores que Influenciam o Consumidor na Escolha de Restaurantes do Tipo Self Service. Revista Contextos da Alimentação, v. 3, n. 1, p. 3-17, 2014.

BLANGER, L. D; NUNES, M. R. S; SANT'ANNA. V. Avaliação das condições higiênico-sanitárias dos serviços de alimentação com Self-service de Encantado - RS. Revista eletrônica científica UERGS, v. 3, n. 1, p. 194-214, 2017.

BRASIL. MINISTÉRIO DA SAÚDE/SVS/SINAN Doenças Transmitidas por Alimentos. Brasília, 2015. Disponível em: http://u.saude.gov.br/images/pdf/2015/novembro/09/Apresenta-o-dadosgerais-DTA-2015.pdf.Acesso em: 30 set. 2020.

BRASIL. MINISTÉRIO DA SAÚDE ANVISA. Resolução nº RDC 216, 15 de setembro de 2004. Dispõe sobre Regulamento Técnico de Boas Práticas para Serviços de Alimentação. Diário Oficial [da República Federativa do Brasil]. Brasília, 16 set. 2004.

BRASIL. Ministério da Saúde. Manual Integrado de Prevenção e Controle deDoenças Transmitidas por Alimentos. Brasília: Secretaria de Vigilância em Saúde, 2001. 136 p.

BRASIL. Portaria n. 1428, de 26 de novembro de 1993. Dispõe sobre o controle de qualidade na área de alimentos. Diário Oficial da União, Brasília, 2 de dezembro de 1993.

CARELlE, A. C; CÂNDIDO, C. C. Manipulação e higiene dos alimentos. $2^{\circ}$ ed. Editora Érica Ltda. São Paulo, 2014.

COSTACURTA. F.A.D; PEREIRA, G.S; VASQUES, C. T. A importância do profissional de nutrição na atuação em unidades de alimentação e nutrição comerciais e industriais. In: $7^{\circ}$ Simpósio de Segurança alimentar. Inovação com sustentabilidade, 2020, Porto Alegre-RS, Brasil. Anais [...] Porto Alegre-RS, 2020. Disponível em: http://schenautomacao.com.br/ssa7/anais/trabalhos.php. Rio grande do Sul, RS, 2020. Acesso em: 22 fev. 2021.

FIDELIS, J. C.; MOURA, M. M.; SANTANA, G. A.; SALVESTRO, A. C.; MADRONE, G. S.; TERRA, C. O. Elaboração e Implantação de Procedimentos Operacionais Padrão no Setor de Laticínios. Revista Tecnológica, v. 19, n.1, p. 75-78, 2010.

JOMORI, M. M. Escolha alimentar do comensal de um restaurante por peso. 2006. $141 \mathrm{f}$. Dissertação (Mestrado em Nutrição) Universidade Federal de Santa Catarina, Florianópolis, SC, 2006.

JUNIOR, G. N. S. A relevância do responsável técnico nutricionista na prevenção de surtos alimentares em unidades de alimentação e nutrição. Brazilian Journal of Devolompment, v. 6, n. 10, p. 77795-77807, 2020.

KETZER, L. T. B. Dificuldades encontradas por nutricionistas na aplicação de boas práticas. Trabalho de Conclusão de Curso (Bacharel em Nutrição) - Universidade Regional do Noroeste do Estado do Rio Grande do Sul - UNIJUÍ, Ijuí, RS. 2013.

LENZ, B. E; BACKES, J; BERTANI, J. P. B; FASSINA, P. Verificação de boas práticas em duas Unidades de Alimentação e Nutrição inseridas em dois municípios do Rio Grande do Sul. Revista Simbio-Logias, v.11, n. 15, p. 62-76, 2019. 
MACIEL, A. R; OLIVEIRA, J. B. H. S.G; MEIRELES, N. M. S; SILVA, I. S; NASCIMENTO, M; SILVA, L. L; ALMEIDA, B. S. Verificação das boas práticas de fabricação em panificadoras da cidade de Marabá, Pará, Brasil. Scientia Plena, v. 12, n. 06, p. 1-6, 2016.

MAIA, M. O; MAIA, M. O. Avaliação das condições higiênico-sanitárias de uma lanchonete no município de Limoeiro do Norte - CE. Revinter, v. 10, n. 01, p. 45-56, 2017.

MELO, J. C.; CRUZ, N. T. S.; BESERRA, M. L. S. Segurança Alimentar em Restaurantes de Teresina - PI. Revista Interdisciplinar, v. 7, n. 2, p. 60-69, 2014.

MONTEIRO, M. A. M.; FRÓES, J. A. C; FONTES, R. B.R; RIBEIRO, R. C. Qualidade na Produção de Refeições em Restaurantes do tipo Self Service. Demetra, v. 9, n.4, p. 955-961, 2014.

NUNES, S. M.; CERGOLE-NOVELLA, M. C; TIBA, M. R; ZANON, C. A; BENTO, I. S.S; PASCHUALINOTO, A. L; THOMAZ, I; SILVA, A. A; WALENDY, C. H. Surto de doença transmitida por alimentos nos municípios de Mauá e Ribeirão Pires - SP. Revista Higiene Alimentar, v. 31, n. 245/246, p. 97-102, 2017.

RIO GRANDE DO SUL. Portaria no 78, de 30 de janeiro de 2009. Aprova a Lista de Verificação em Boas Práticas para Serviços de Alimentação, aprova Normas para Cursos de Capacitação em Boas Práticas para Serviços de Alimentação e dá outras providências. Secretaria da Saúde. Porto Alegre, 30 jan. 2009. Disponível em: http://www.tramandai.rs.gov.br/download/portaria estadual_078_2009.pdf.

SANTOS, R. M. S; GOUVEIA, D. S; ROCHA, A. P. T; SILVA, W. M; LINS, A. D. F. Avaliação de restaurante universitário por meio do regulamento técnico de boas práticas para serviços de alimentação. Revista Verde, v. 10, n. 2, p. 26-32, 2015.

SILVA, A. A; BASSANI, L; RIELLA, C. O; ANTUNES, M. T. Manipulação de alimentos em uma cozinha hospitalar: ênfase na segurança dos alimentos. Caderno Pedagógico, v. 12, n. 1, p. 111-123, 2015.

SILVEIRA, J. T; BRASIL, C. C. B; FLORIANO, J. M; SCHWARZER, P. F. Condições higiênicas e boas práticas de manipulação em serviços de alimentação da cidade de Itaqui - RS. Revista Visa em debate sociedade, ciência e tecnologia, v. 3, n. 2, p. 144-149, 2015.

STANGARLIN, F. L.; SERAFIM, A. L.; SACOOL, A. L de F. Instrumentos para elaboração do Manual de Boas Práticas e dos Procedimentos Operacionais Padronizados em serviços de alimentação. Rio de Janeiro: Rubio, 2016.

STOFFEL, F; BARRETO, L. T. P. Avaliação de boas práticas em restaurante especializado em culinária oriental. Revista Higiene Alimentar, v. 32, n. 276/279, p. 860-864, 2018.

TEIXEIRA, S; CARVALHO, Z. M. J; BISCONTINI, T. M. Administração Aplicada as Unidades de Alimentação e Nutrição. 2. ed. São Paulo: Atheneu, 1997. 230 p. 